\title{
Thermoeconomic optimization of absorption chiller cycle
}

\author{
H. Mashayekh ${ }^{1}$, G.R. Salehi ${ }^{2} *$ E. Taghdiri ${ }^{3}$, M.H. Hamedi ${ }^{3}$ \\ ${ }^{1}$ Islamic Azad University Science \& Research Branch, Tehran, Iran \\ ${ }^{1}$ Islamic Azad University Nowshahr Branch, Nowshahr, Iran \\ ${ }^{2}$ KNToosi University of Technology, Tehran, Iran \\ * Corresponding author. Tel: +989122031671, E-mail: rezasalehi20@gmail.com
}

\begin{abstract}
In this paper, absorption chillers are modeled as four heat sources: the generator, the evaporator, the condenser, and the absorber and thermoeconomic issues are examined using the available relations. In order to simplify the calculations, all processes are assumed to be reversible. Since heat exchangers are expensive facilities, therefore, reducing the total heat transfer area is taken as the design criterion. In this paper, first the thermoeconomic criterion, taken as the total cost of unit of refrigeration which includes the capital cost and the energy cost, is defined. In the following, the available relationships are used to calculate the maximum value of the thermoeconomic criterion and the maximum refrigeration load. Next, optimal working conditions are specified for absorption chillers and after that, the effect of the thermoeconomic parameter on the maximum thermoeconomic criterion, coefficient of performance and the specific refrigeration load corresponding to the maximum value of the thermoeconomic criterion are investigated.
\end{abstract}

Keywords: thermoeconomic performance, absorption chiller, optimization

\section{Introduction}

Nowadays, costs of energy consumption and electricity compared to those of fossil fuels have caused engineers in most countries to consider using absorption chillers instead of compression chillers. However, fuel costs need to be controlled in some way and consequently, economical studies are carried out constantly on opt imization of absorption chillers and reducing their economical costs.

A new criterion has been used in the recent years to evaluate the degree of optimality of thermodynamic cycles. The thermoeconomic criterion economically investigates the phenomena. Capital costs and energy costs are considered in these investigations using thermodynamic relationships and working conditions are designed in a way that the operation is economically optimum. Chen and Schouten (1998) evaluated the optimal value of coefficient of performance in irreversible absorption chiller systems [1]. Next, Chen in 1999 evaluated the optimal value of coefficient of performance for the irreversible 4-heat exchange-surface absorption chiller in the maximum specific cooling load [2]. This was followed by other researches with the aim of optimization of absorption chiller processes and other processes similar to those, including thermoeconomic optimization of the reversible sterling heat pump cycle by Tyagi, Chena, and Kaushikb[3].

The largest portion of capital cost of absorption chiller pertains to the heat exchangers used in the generator, condenser, evaporator, and the absorber.

\section{Modeling of the cycle and its relationships}

In order to simplify the calculations, absorption chiller cycle is assumed to consist of four heat sources including the absorber, the generator, the evaporator, and the condenser, as illustrated in figure 1. We assume that the processes take place reversibly. It is also assumed that the flow is constant in working parts of the cycle and heat transfer between the elements and heat sources during the time of a complete cycle $\tau$, happen at temperatures $T_{a}, T_{c}, T_{e}, T_{g}$, respectively. There are thermal resistances between the external components and the 
operating elements of the cycle. Temperatures of working components in the generator, evaporator, condenser and the absorber are respectively $T_{4}, T_{3}, T_{2}, T_{1}$. Heat transfer coefficients are accordingly $U_{4}, U_{3}, U_{2}, U_{1}$. Moreover, heat transfer surfaces are $A_{4}, A_{3}, A_{2}, A_{1}$, in the generator, the evaporator, the condenser and the absorber, respectively. The work input for the pump in the cycle Is taken as negligible compared to the heat input to the generator.

It is assumed that the heat transfer between the operating components of the cycle and the external heat sources follows the linear (Newtonian) heat transfer mode and the four processes take place isothermally. Thus, heat transfer equations of all the four processes are written as follows:

$$
\begin{aligned}
& Q_{1}=U_{1} A_{1}\left(T_{g}-T_{1}\right) \tau \\
& Q_{2}=U_{2} A_{2}\left(T_{e}-T_{2}\right) \tau \\
& Q_{3}=U_{3} A_{3}\left(T_{3}-T_{c}\right) \tau \\
& Q_{4}=U_{4} A_{4}\left(T_{4}-T_{a}\right) \tau
\end{aligned}
$$

Where $Q_{4}, Q_{3}, Q_{2}, Q_{1}$ are heat transfers in the generator, the evaporator, the condenser and the absorber, respectively, From the first law of thermodynamic we have:

$$
Q_{1}+Q_{2}-Q_{3}-Q_{4}=0
$$

Considering the second law of thermodynamic and the reversibility of the cycle we

have: $\frac{Q_{1}}{T_{1}}+\frac{Q_{2}}{T_{2}}-\frac{Q_{3}}{T_{3}}-\frac{Q_{4}}{T_{4}}=0$

Since the heat exchangers are the expensive component in the cycle, reduction of the heat transfer area (A) is taken as the design criterion. Therefore, by optimizing the total heat transfer area we can obtain optimality.

$$
A=A_{1}+A_{2}+A_{3}+A_{4}
$$

The parameter (a) is defined as the total distribution rate between the condenser and the absorber:

$$
a=\frac{Q_{3}}{Q_{4}}
$$

According to the standard definitions of coefficient of performance (COP) and specific cooling load (r) and equations (1) to (8), coefficient of performance can be obtained as follows:

$$
\varepsilon=\frac{Q_{2}}{Q_{1}}=\frac{T_{4}^{-1}+a T_{3}^{-1}-(1+a) T_{1}^{-1}}{(1-a) T_{2}^{-1}-T_{4}^{-1}-a T_{3}^{-1}}
$$

And the specific cooling load is obtained as follows: 


$$
\begin{aligned}
& r=\frac{Q_{2}}{A \tau}=\left\{\frac{1}{U_{2}\left(T_{e}-T_{2}\right)}+\frac{T_{2}^{-1}-T_{4}^{-1}+a\left(T_{2}^{-1}-T_{3}^{-1}\right)}{U_{1}\left(T_{g}-T_{1}\right)\left[T_{4}^{-1}-T_{1}^{-1}+a\left(T_{3}^{-1}-T_{1}^{-1}\right)\right]}\right. \\
& \left.+\left[\frac{1}{U_{4}\left(T_{4}-T_{a}\right)}+\frac{a}{U_{3}\left(T_{3}-T_{c}\right)}\right] \frac{T_{2}^{-1}-T_{1}^{-1}}{T_{4}^{-1}-T_{1}^{-1}+a\left(T_{3}^{-1}-T_{1}^{-1}\right)}\right\}^{-1}
\end{aligned}
$$

Equations 9 and 10 are the general relationships for absorption chillers with four reversible heat sources. These relationships can be used to obtain thermoeconomical optimum performance for this type of absorption chillers. According to the studies carried out by Kodal and Shahin as given in [5], the thermoeconomic criterion for absorption chillers with four reversible heat sources is defined as the total price of unit cooling load which includes both capital and energy costs. Therefore, the function which is to be optimized is as follows:

$$
F=\frac{\frac{Q_{2}}{\tau}}{C_{i}+C_{e}}
$$

Where $C_{i}, C_{e}$ are capital and energy costs in the unit of time, respectively. The capital cost of absorption chillers is assumed to be proportional to its total heat transfer area:

$$
C_{i}=k_{1} A
$$

Where $k_{1}$ is the capital recovery factor, the capital cost for the unit heat transfer area. Energy consumption cost is directly proportional to the rate of heat input:

$$
C_{e}=k_{2} \frac{Q_{1}}{\tau}
$$

Where $k_{2}$ is the unit cost of energy. By substituting equations 12 and 13 in 11, we obtain:

$$
F=\frac{Q_{2} / \tau}{\left(k_{1} A+k_{2} Q_{1} / \tau\right)}=\left(k_{1} r^{-1}+k_{2} \varepsilon^{-1}\right)^{-1} \text {. }
$$

By defining $k=\frac{k_{1}}{k_{2}}$ as the thermoeconomic parameter having the dimension $\frac{K W}{m^{2}}$, when the capital cost increases and the cost of energy consumption reduces, the thermoeconomic parameter $k$ goes up. The optimal relationship for the total refrigeration load and the COP of absorption chillers is as follows:

$$
\begin{aligned}
r= & U_{2}\left[T_{\mathrm{c}} T_{\mathrm{c}}+a T_{\mathrm{e}} T_{\mathrm{a}}-\left(T_{\mathrm{g}} \varepsilon+T_{\mathrm{c}}\right) \frac{T_{\mathrm{c}} T_{\mathrm{a}}(1+a)}{T_{\mathrm{g}}(1+\varepsilon)}\right] \\
& \times\left\{\left[\left(1+b_{2}\right)^{2} T_{\mathrm{c}}+a\left(1+b_{3}\right)^{2} T_{\mathrm{a}}-\frac{a}{1+a}\left(b_{2}-b_{3}\right)^{2} T_{\mathrm{c}}\right]-\left(1-b_{1}\right)^{2} \frac{(1+a) T_{\mathrm{a}} T_{\mathrm{c}}}{(1+\varepsilon) T_{\mathrm{g}}}\right. \\
& \left.+\frac{T_{\mathrm{e}}}{\varepsilon T_{\mathrm{g}}}\left[\left(b_{1}+b_{2}\right)^{2} T_{\mathrm{c}}+a\left(b_{1}+b_{3}\right)^{2} T_{\mathrm{a}}-\frac{a}{1+a}\left(b_{2}-b_{3}\right)^{2} T_{\mathrm{g}}\right]\right\}^{-1},
\end{aligned}
$$


Where: $b_{1}=\sqrt{\frac{U_{2}}{U_{1}}}$

By combining (14) and (15), the ration of the optimal value of the thermoeconomic criterion and the COP of absorption chillers with four reversible heat sources is obtained as follows:

$$
\begin{aligned}
k_{2} F= & \left\{\frac{k}{U_{2}}\left[T_{\mathrm{e}} T_{\mathrm{c}}+a T_{\mathrm{e}} T_{\mathrm{a}}-\left(T_{\mathrm{g}} \varepsilon+T_{\mathrm{e}}\right) \frac{T_{\mathrm{c}} T_{\mathrm{a}}(1+a)}{T_{\mathrm{g}}(1+\varepsilon)}\right]^{-1}\right. \\
& \times\left[\left(1+b_{2}\right)^{2} T_{\mathrm{c}}+a\left(1+b_{3}\right)^{2} T_{\mathrm{a}}-\frac{a}{1+a}\left(b_{2}-b_{3}\right)^{2} T_{\mathrm{e}}\right]-\left(1-b_{1}\right)^{2} \frac{(1+a) T_{\mathrm{a}} T_{\mathrm{c}}}{(1+\varepsilon) T_{\mathrm{g}}} \\
& \left.+\frac{T_{\mathrm{c}}}{\varepsilon T_{\mathrm{g}}}\left[\left(b_{1}+b_{2}\right)^{2} T_{\mathrm{c}}+a\left(b_{1}+b_{3}\right)^{2} T_{\mathrm{a}}-\frac{a}{1+a}\left(b_{2}-b_{3}\right)^{2} T_{\mathrm{g}}\right]+\varepsilon^{-1}\right\}^{-1} .
\end{aligned}
$$

Equation 19 gives the optimum thermoeconomic criterion for a given value of COP and also the optimum coefficient of performance for a given thermoeconomic parameter in reversible absorption chillers. Using equation19, we can evaluate other characteristics of thermoeconomic operation of reversible absorption chillers which obey the linear (Newtonian) heat transfer law.

Equation 19 demonstrates that for the thermoeconomic parameter we have $F=0$ when $\varepsilon=0$ or $\varepsilon=\varepsilon_{c}$, where:

$$
\varepsilon_{\mathrm{c}}=\frac{T_{\mathrm{a}}^{-1}+a T_{\mathrm{c}}^{-1}-(1+a) T_{\mathrm{g}}^{-1}}{(1+a) T_{\mathrm{e}}^{-1}-T_{\mathrm{a}}^{-1}-a T_{\mathrm{c}}^{-1}},
$$

is the coefficient of performance for an absorption chiller with four reversible heat sources.

When $\varepsilon<\varepsilon_{c}$, the maximum thermoeconomic criterion is derived. Using (19) and the final condition $\frac{d\left(k_{2} F\right)}{d \varepsilon}=0$, we can evaluate $\operatorname{COP}\left(\varepsilon_{r}\right)$ for the maximum value of the maximum thermoeconomic criteria $\left(F_{\max }\right)$ :

$$
\varepsilon_{F}=\left[1-\sqrt{1-\frac{(1+a) T_{\mathrm{g}}^{-1}-T_{\mathrm{a}}^{-1}-a T_{\mathrm{c}}^{-1}}{T_{\mathrm{a}}^{-1}+a T_{\mathrm{c}}^{-1}-(1+a) T_{\mathrm{e}}^{-1}}} d_{1}\right] d_{1}^{-1}
$$

Where

$$
\begin{aligned}
d_{1}= & \frac{(1+a)\left(T_{\mathrm{g}}-T_{\mathrm{e}}\right) d_{2}}{T_{\mathrm{e}}^{2}\left[T_{\mathrm{a}}^{-1}+a T_{\mathrm{c}}^{-1}-(1+a) T_{\mathrm{e}}^{-1}\right]\left\{d_{3}+\left[T_{\mathrm{c}} T_{\mathrm{g}}+a T_{\mathrm{a}} T_{\mathrm{g}}-(1+a) T_{\mathrm{a}} T_{\mathrm{c}}\right] U_{2} / k\right\}} \\
& +\frac{\left(1-b_{1}\right)^{2}(1+a) T_{\mathrm{a}} T_{\mathrm{c}}-d_{3} T_{\mathrm{e}}-\left[T_{\mathrm{e}} T_{\mathrm{c}}+a T_{\mathrm{e}} T_{\mathrm{a}}-(1+a) T_{\mathrm{a}} T_{\mathrm{c}}\right] T_{\mathrm{g}} U_{2} / k}{T_{\mathrm{e}}\left\{d_{3}+\left[T_{\mathrm{c}} T_{\mathrm{g}}+a T_{\mathrm{a}} T_{\mathrm{g}}-(1+a) T_{\mathrm{a}} T_{\mathrm{c}}\right] U_{2} / k\right\}} \\
d_{2}= & \left(1+b_{2}\right)^{2} T_{\mathrm{c}}+a\left(1+b_{3}\right)^{2} T_{\mathrm{a}}-\left(b_{2}-b_{3}\right)^{2} T_{\mathrm{e}} a /(1+a) \\
d_{3}= & \left(b_{1}+b_{3}\right)^{2} T_{\mathrm{c}}+a\left(b_{1}+b_{3}\right)^{2} T_{\mathrm{a}}-\left(b_{2}-b_{3}\right)^{2} T_{\mathrm{g}} a /(1+a)
\end{aligned}
$$


Substituting (21) in (19), we can obtain the maximum thermoeconomic limit for a reversible absorption chiller. Substituting (21) in (15), we can obtain the specific refrigeration load $\left(r_{F}\right)$ for the maximum thermoeconomic criterion. Three parameters $F_{\max }, r_{F}, \varepsilon_{F}$ are important for the optimum design of reversible absorption chillers. These parameters result in the lowest value of COP and the lowest value of the characteristic refrigeration load and the top limit of the thermoeconomic criterion $[5,6,7]$.

\section{Investigation and conclusion}

In order to examine the thermoeconomic of the cycle and the impact of its different parameters, a cas e study was analyzed and the obtained circumstances were compared with each other. The following data were known for the aforementioned case study.

$T_{g}=403 K, T_{e}=293 K, T_{c}=313 K, T_{a}=305 K, a=1, U_{1}=U_{2}=U_{3}=U_{4}=0.5 \frac{K W}{K \cdot m^{2}}$

By changing any of the following parameters, we take other parameters as constant and equal to the values mentioned above. The characteristic charts of the problem at hand were obtained using the above data. Figure 2 shows the thermoeconomic criteria versus the coefficient of performance of a reversible chiller with $k=1 \frac{K W}{m^{2}}$. The value of the maximum thermoeconomic criterion $\left(F_{\max }\right)$ can be obtained from this curve. As you can see, this chart consists of two sections with negative and positive slopes. The part which has a negative slope represents the optimum region for operation of absorption chillers. In figure 3, variations of the thermoeconomic criterion in terms of the characteristic refrigeration load is shown. Here too, we have $k=1 \frac{K W}{m^{2}}$. From this curve one can easily obtain the maximum value for the thermoeconomic criterion $\left(F_{\max }\right)$ and the maximum refrigeration load $\left(r_{\max }\right)$. This curve has three parts. It is obvious that the optimum working conditions for absorption chillers are in the region with negative slope.

Figures 2 and $3 \mathrm{can}$ help one find the optimum region for operation of absorption chillers, which are the regions with negative slopes. This region should abide by the following conditions:

$$
F_{r} \leqslant F \leqslant F_{\max } \quad(25) \quad \varepsilon_{F} \leqslant \varepsilon \leqslant \varepsilon_{r} \quad \text { (26) } \quad r_{F} \leqslant r \leqslant r_{\max }
$$

Where $F_{r}$ the thermoeconomic criterion for the maximum refrigeration is load $\left(r_{\max }\right)$ and $\varepsilon_{r}$ is the COP for the maximum value of characteristic refrigeration load for the reversible absorption chiller which can be obtained from the following equation:

$$
\varepsilon_{r}=\left[1-\sqrt{1-\frac{(1+a) T_{\mathrm{g}}^{-1}-T_{\mathrm{a}}^{-1}-a T_{\mathrm{c}}^{-1}}{T_{\mathrm{a}}^{-1}+a T_{\mathrm{c}}^{-1}-(1+a) T_{\mathrm{c}}^{-1}}} d_{4}\right] d_{4}^{-1}
$$

Where:

$$
d_{4}=\frac{(1+a)\left\{\left(1-b_{1}\right)^{2}\left[T_{\mathrm{e}} T_{\mathrm{c}}+a T_{\mathrm{e}} T_{\mathrm{a}}-(1+a) T_{\mathrm{a}} T_{\mathrm{c}}\right]+\left(T_{\mathrm{g}}-T_{\mathrm{e}}\right) d_{2}\right\}}{T_{\mathrm{e}}^{2}\left[T_{\mathrm{a}}^{-1}+a T_{\mathrm{c}}^{-1}-(1+a) T_{\mathrm{e}}^{-1}\right] d_{3}}-1
$$


Substituting 28 in 19, we can obtain the maximum value for the characteristic refrigeration load. Moreover, by substituting 28 in (15), we can obtain the value of the thermoeconomic criterion for the maximum characteristic refrigeration load, $\left(F_{r}\right)$. The values of the parameters which influence the operation of the cycle are changed and their effects on the cycle are compared. Figure 4 shows the characteristic curve of the thermoeconomic criterioncoefficient of performance for four different values of the thermoeconomic criterion $(k)$ and figure 5 shows the characteristic curve of the thermoeconomic criterion - refrigeration load for the same four values of the thermoeconomic criterion. We can deduce from these curves that the optimum thermoeconomic criterion for a known value of COP, the thermoeconomic criterion for a specific capacity of refrigeration and optimum coefficient of performance and characteristic refrigeration capacity for a known value of the thermoeconomic criterion, all reduce by increasing the thermoeconomic parameter $(k)$. Figure 6 shows the characteristic thermoeconomic parameter- coefficient of performance curve for four different values of total rate of distribution on heat output $(a)$ and figure 7 shows the characteristic thermoeconomic criterion-refrigeration load curve for the same four values of the total rate of heat output.

It can be deduced from these curve that the optimum thermoeconomic criterion for a known value of COP, the thermoeconomic criterion for a special refrigeration capacity and the optimum coefficient of performance, and the characteristic refrigeration performance for a known value of thermoeconomic criterion all decrease by increasing the total heat output distribution rate $(a)$. As it is evident from figure $7, \mathrm{t}$ he maximum value for the thermoeconomic criterion and its corresponding coefficient of performance, reduce by increasing the thermoeconomic parameter $(k)$ and the specific refrigeration load corresponding to the maximum thermoeconomic criterion increases by increasing the thermoeconomic parameter $(k)$.

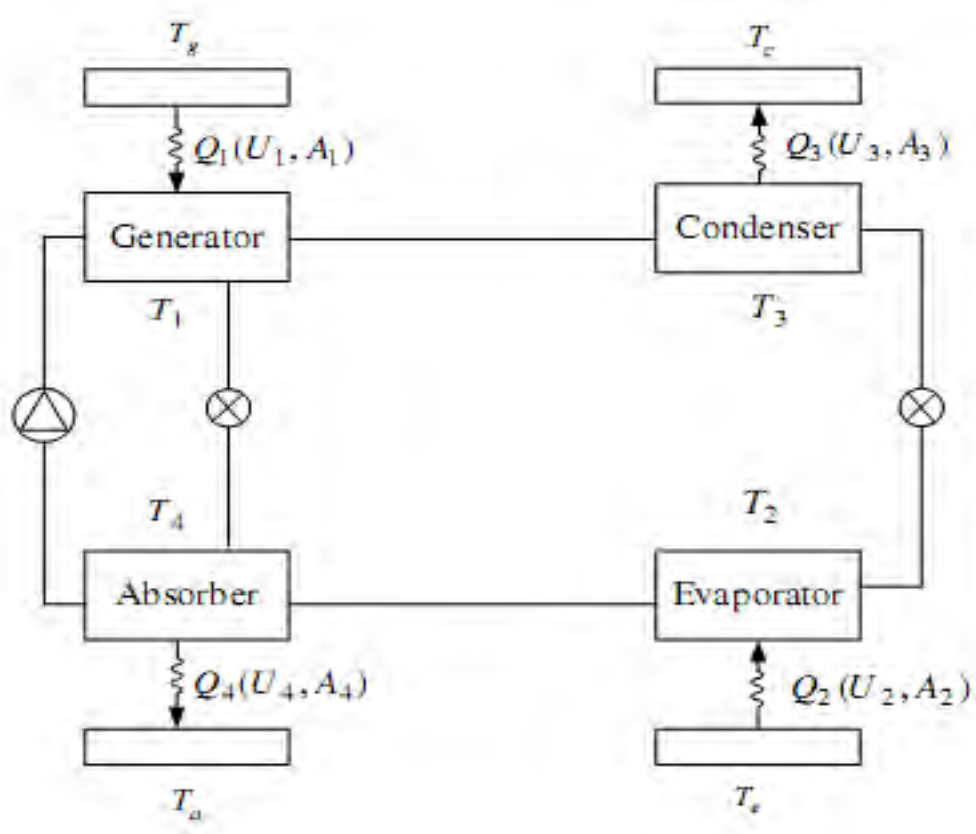

Fig 1: modeling of the cycle 


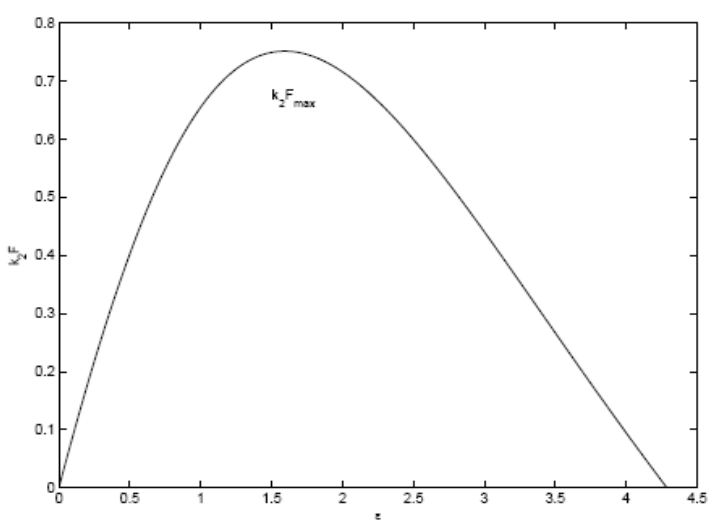

Figure 2: the thermoeconomic criterion in terms of coefficient of performance

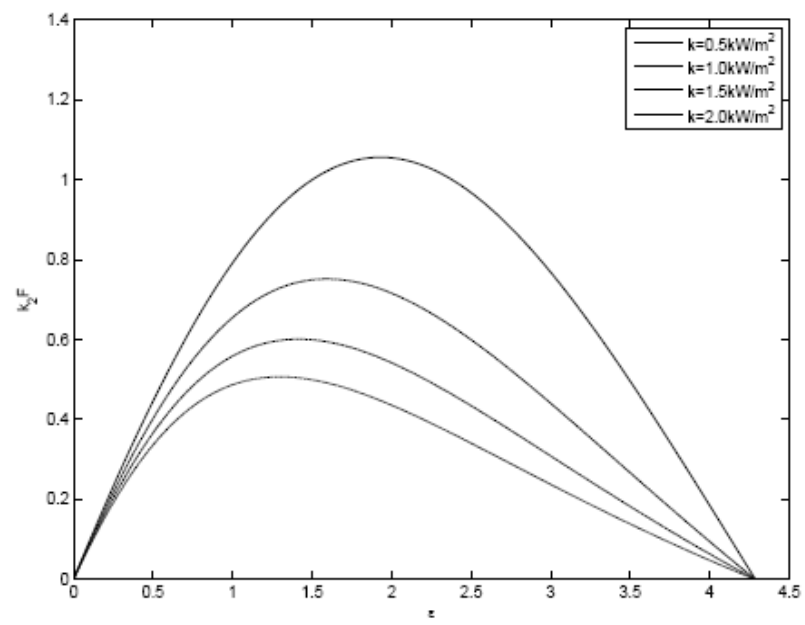

Figure 4: the effect of the thermoeconomic parameter on the ration of the thermoeconomic criterion and the coefficient of performance

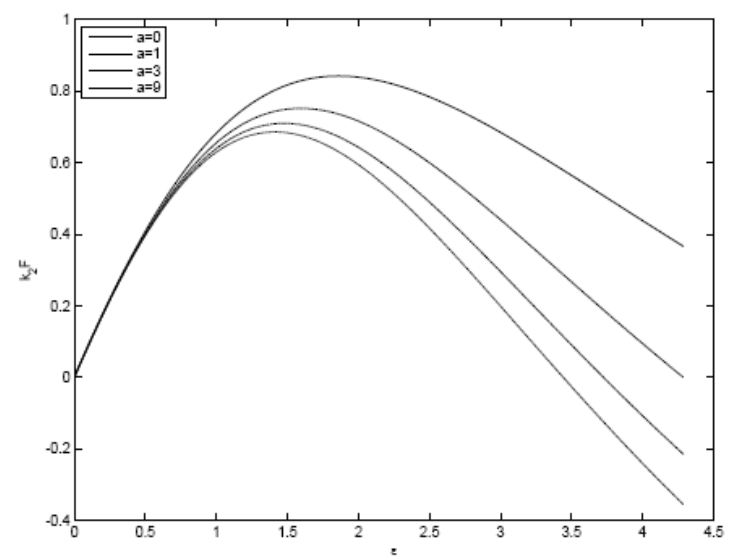

Figure 6: the effect of the total rate of heat output on the ratio of the thermoeconomic criterion and coefficient of performance

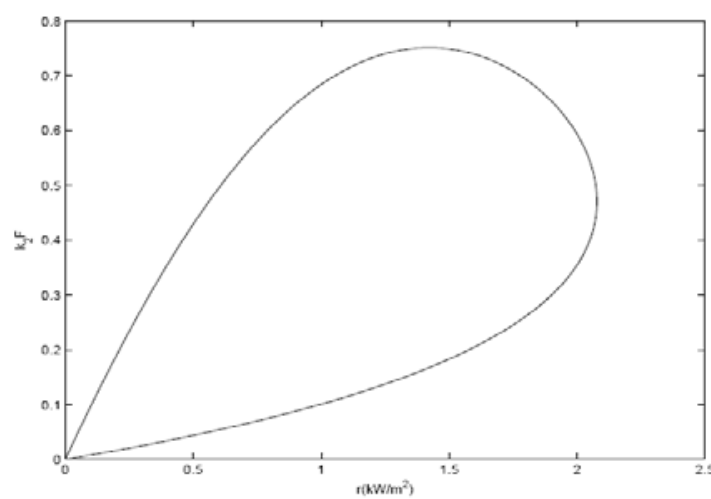

Figure 3: the curve of the thermoeconomic criterion in terms of the characteristic curve of the system.

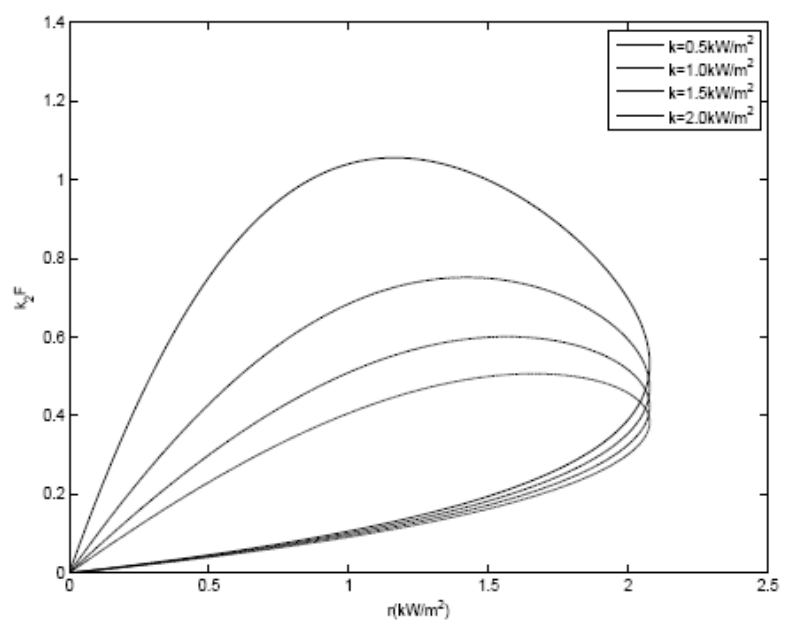

Figure 5: the effect of the thermoeconomic parameter on the thermoeconomic criterion and refrigeration capacity

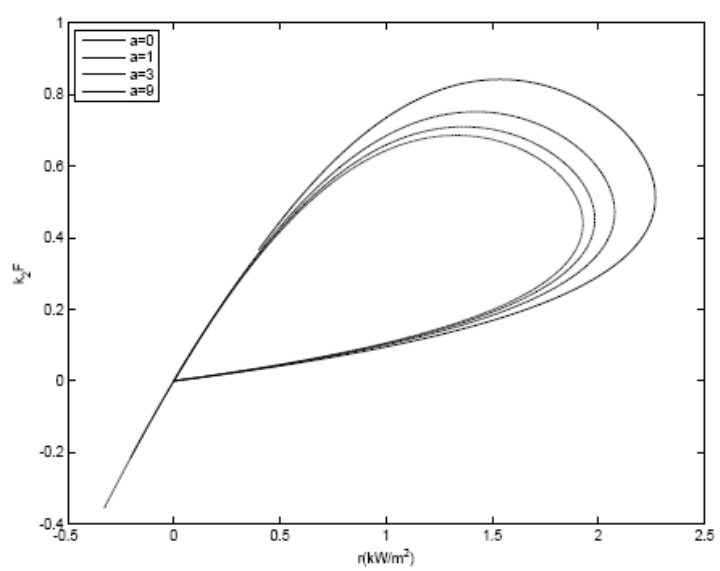

Figure 7: the effect of the total rate of heat output on the ratio of the thermoeconomic criterion and refrigeration capacity 


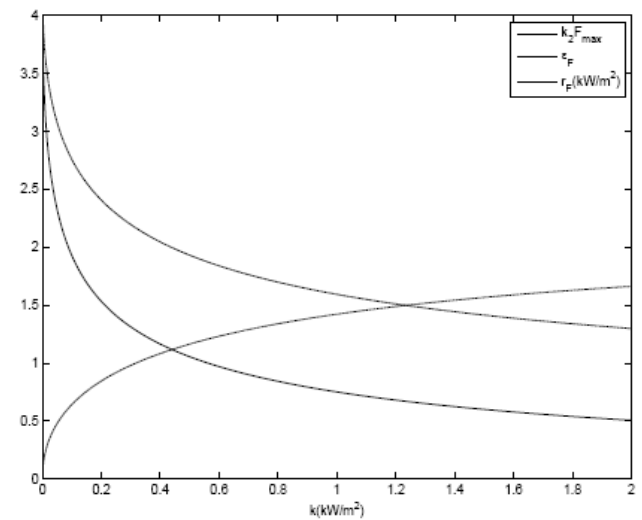

Figure 8: the effect of the thermoeconomic parameter on $F_{\max }, r_{F}, \varepsilon_{F}$

\section{Conclusion}

In this paper, the performance of absorption chillers with four reversible heat sources was analyzed and optimized with the existing relationships. The range of the important parameters for the absorption cooling cycle with four reversible heat sources for its optimum operation was determined. The obtained results can be used as a theoretic guide for further studies on the thermoeconomic optimization and further development of performance of absorption chiller cycles.

\section{References}

[1] Chen J, Schouten "A. Optimal performance-characteristic of an irreversible absorptionrefrigerationsystem”. Energy Convers Manage 1998

[2] Chen J. "The optimal performance-characteristic of a four-temperature-level irreversible absorption-refrigerator at maximum specific-cooling load”. J Phys D: Appl Phys 1999

[3] S.K. Tyagi, Jincan Chena, S.C. Kaushikb "Thermoeconomic optimization and parametric study of an irreversible Stirling heat pump cycle"., International Journal of Thermal Sciences 43

[4] Wang S.K., "Handbook of Air Conditioning and Refrigeration" McGraw-Hill, 2nd Ed, New York, 2000.

[5] Kodal A, Sahin B, Ekmekci I, Yilmaz T. "Thermoeconomic optimization for irreversible absorption-refrigeration and heat pumps". Energy Convers Manage 2003.

[6] Sahin B, Kodal A. "Finite-time thermoeconomic optimization for endoreversible refrigerators and heat pumps." Energy Convers Manage 1999;40(9):951-60.

[7] Chen L, Sun F, Chen W, Wu C. "Optimal performance coeffcient and cooling-load relationship of a three-heat-reservoir endoreversible refrigerator. "Int J Power Energy Sys 1997; 17(3):206-8. 